\title{
Pediatric mercury poisoning, brain MRI, and white matter hyperintensities
}

\author{
Antonella Costa $\cdot$ Vincenzo Branca Paolo D. Pigatto • \\ Gianpaolo Guzzi
}

Received: 10 January 2011 / Accepted: 3 February 2011 /Published online: 23 February 2011

(C) Springer-Verlag 2011

\section{Dear Editor,}

In their valuable article of the first description in the literature of inorganic mercury salt poisoning associated with white matter lesions (WMLs) on $\mathrm{T}_{2}$-weighted MR images of the brain, Benz et al. [1] describe a 4-year-old girl with a nonfatal percutaneous inorganic mercury intoxication due to topical application of mercurycontaining skin lightening cream. The authors are to be commended on their case report as they also provide an excellent discussion of the effects of chelation therapy with 2,3-dimercapto-1-propanesulfonic acid. In the "Introduction section" of their case report, Benz et al. state that, "Data about magnetic resonance imaging (MRI) findings in intoxication from inorganic $\mathrm{Hg}$ did not exist up to now" [1].

We wonder if they are aware that diffuse and punctiform subcortical WMLs on $\mathrm{T}_{2}$-weighted MR images in both frontal region-including cortical atrophy-have been observed previously by neurotoxicologists in a thermometer factory worker who had been chronically exposed to

Vincenzo Branca deceased during manuscript preparation.

A. Costa $\cdot$ V. Branca

Department of Diagnostic and Interventional Neuroradiology,

IRCCS Maggiore Hospital, Mangiagalli and R. Elena Foundation,

University of Milan,

Milan, Italy

P. D. Pigatto

Department of Technology for Health, Dermatological Clinic,

IRCCS Galeazzi Hospital, University of Milan,

Milan, Italy

G. Guzzi $(\bowtie)$

Italian Association for Metals and Biocompatibility

Research - A.I.R.M.E.B,

Via A. Banfi, 4,

20122 Milan, Italy

e-mail: gianpaolo_guzzi@fastwebnet.it inorganic mercury vapor [3]. We also wish to highlight the important finding in the case study of Benz et al. [1] that overexposure to inorganic mercury salts was associated with elevated serum neuron-specific enolase (NSE) levels in response to mercury-induced neurotoxic injury, following damage to neuronal membranes. This observation is very interesting and concurs with our own anecdotal experiences. Bichloride of mercury (corrosive sublimate) has also been reported to induce and increase the expression of NSE in vitro. Thus, under in vitro culture conditions, NSE has been used to assess the inorganic mercury neurotoxicity [2]. We encourage investigators in future studies of clinical toxicity of mercury to explore this interaction and include measures of serum NSE levels, especially in patients with inorganic mercury overexposure.

Conflicts of interest We have no conflicts of interest connected with this work.

Funding None.

\section{References}

1. Benz MR, Lee SH, Kellner L, et al. (2011) Hyperintense lesions in brain MRI after exposure to a mercuric chloride-containing skin whitening cream. Eur J Pediatr Oct 30. doi:10.1007/s00431-010-1333-1

2. Huang J, Tanii H, Kato K et al (1993) Neuron and glial cell marker proteins as indicators of heavy metal-induced neurotoxicity in neuroblastoma and glioma cell lines. Arch Toxicol 67:491-6

3. White RF, Feldman RG, Moss MB, Proctor SP (1993) Magnetic resonance imaging (MRI), neurobehavioral testing, and toxic encephalopathy: two cases. Environ Res 61(1):117-23 